\title{
TWITTER BASED SENTIMENT ANALYSIS OF IMPACT OF COVID-19 ON EDUCATION GLOBALY
}

\author{
Swetha Sree Cheeti, Yanyan Li and Ahmad Hadaegh \\ California State University-San Marcos, San Marcos, California, USA
}

\begin{abstract}
Education system has been gravely affected due to widespread of Covid-19 across the globe. In this paper we present a thorough sentiment analysis of tweets related to education available on twitter platform and deduce conclusions about its impact on people's emotions as the pandemic advanced over the months. Through twitter over ninety thousand tweets have been gathered related to the circumstances involving the change in education system over the world. Using Natural language tool kit (NLTK) functionalities and Naive Bayes Classifier a sentiment analysis has been performed on the gathered dataset. Based on the results of this analysis we infer to exhibit the impact of covid-19 on education and how people's sentiment altered due to the changes with regard to the education system. Thus, we would like to present a better understanding of people's sentiment on education while trying to cope with the pandemic in such unprecedented times.
\end{abstract}

\section{KEYWORDS}

Sentiment Analysis, Education, Covid-19, Tweets, Nä̈ve Bayes Classifier.

\section{INTRODUCTION}

SARS-CoV-2 virus commonly known as Covid-19 has hit the entire world like never known before and became a global threat. Education is the most basic and an important tool to improve one's life. As the world health organization (WHO) has declared Covid-19 as a pandemic, it resulted in near total closure of schools, universities and colleges worldwide. These closures have affected not only students, instructors, staff and, their families but also have a far reaching economic and social consequences [1]. In such a distinctive time, as the world tries to heal and cope, almost every educational organization has moved the education process to remote functioning and virtual learning. Through online classes, using technology students and teachers have been trying to continue the education through various practices as zoom meetings, sharing online materials, recording lectures, power point tutorials and so on. Recent study says that about 1.52 billion learners are affected due to closure of schools, colleges and universities which has shed light on many broader socio-economic issues like student debt, food insecurity, academic integrity, homelessness, childcare, healthcare, housing, internet, digital learning and disability services [1].

In this paper, we attempt to shed light on people's sentiment about education during the global rise of Covid-19. To do this, we use the data from the most extensively used social media platform twitter. Data from social media has been used even in the past to monitor public sentiments and communication during health emergencies [2]. Tweets related to education are collected from twitter and then analyzed using a custom framework to predict the sentiment of each tweet which would give insight about the tweeter sentiment. Based on these results we determine to present a better supportive of people's sentiment on education during the pandemic and through this analysis we could observe and differentiate the level of positive and negative 
implications due to the shift in the education system. Observing which educators and institutions can come up with better efficient ways which might be helpful to smoothen the process of learning. We prospect that with the continuing growth of virtual education monitoring people's sentiment using our enhanced model could make a difference as there is always a scope for betterment to provide the best quality of education in such overwhelming times.

\section{BACKGROUND}

Remote learning has become the new education lifeline and this primary change has an impact on everyone but has more severe consequences for students and their families. To have a successful virtual learning experience, a student should be able to access to a computer and reliable internet which is not the case for every student as some are at a disadvantage of having none. Working parents are at greater disadvantage of missing work hours in order to take care of children at home which indirectly would result in wage loss. Many children across the world rely on schools for free and discounted meals for such children nutrition is especially compromised [1]. Through all these changes students are expected to pay full tuition fee to institutions which is unfair. Learning outcomes cannot be the same with virtual learning as many organizations have to postpone or cancel exams as going ahead with it would lead to violation of social distancing [3]. With many such drawbacks and disadvantages student learning, development and growth are at stake.

Social media is an imperative part of almost every individual's life and is widely used tool for interactions among individuals in which they create, share, and exchange information, ideas and views on topics. Among the social media platforms used today, Twitter is the broadly utilized social media platform and recent study suggests that over 500 million tweets are tweeted each day on twitter. We base our research on tweets collected through such extensively used platform and hope to acquire an insight of people's emotions on changes in education as most people do express their opinions and discuss ongoing issues using social media these days. The data collected through this platform would be useful to analyze, experiment and deduce results to support our research.

\section{RELATED WORK}

Many studies have been conducted earlier using twitter data to predict the sentiment of people in different expanses but to the best of our knowledge there hasn't been a study on people sentiment on education during the pandemic. There are distinctive ways proposed by several researchers to evaluate the sentiments of people using social media. Many of those ways include machine learning algorithms like Naïve Bayes, Max Entropy, Support Vector Machine and so on.

Using multinomial Naïve Bayes method with a training set of tweets that contains only emoticons, Pak and Paroubek [4] presented a model that classifies tweets as positive or negative. But since the training set knowledge is only based on tweets with emoticons, this approach was less effective. Go and L. Huang [5] used both Naïve Bayes and SVM models to analyze twitter data using distant supervision with emoticons in their training data to predict sentiment and determined that SVM performed better. In Agarwal et al. [6] using a tree kernel model tweets are represented as trees and are classified into positive, negative and neutral based on polarity of words with their Part of Speech (POS) tags. A study on sentiment analysis of tweets during covid in Nepal [7] has used python TextBlob library and classified tweets as positive or negative.

There are many different studies conducted to determine the sentiment of tweets, but we propose an enhanced approach which doesn't only rely on emoticons or POS of the tweets to classify a 
International Journal of Artificial Intelligence and Applications (IJAIA), Vol.12, No.3, May 2021

tweet as positive or negative sentiment which is explained in detail in the next section. Further the accuracy attained through our framework also defines the effective performance of our model.

\section{Methodology}

The process to study the impact of covid-19 on education through sentiment analysis of twitter data involves fetching tweets related to education from twitter and then applying Natural language tool kit (NLTK) functionalities to clean the data and split the data into tokens for accurate assessment. Later this modified data is passed through a designed framework for analysis and is trained using Naive Bayes classifier to study each tweet and predict the sentiment of the tweeter. A detailed explanation of steps involved in this research is given in the following sections.

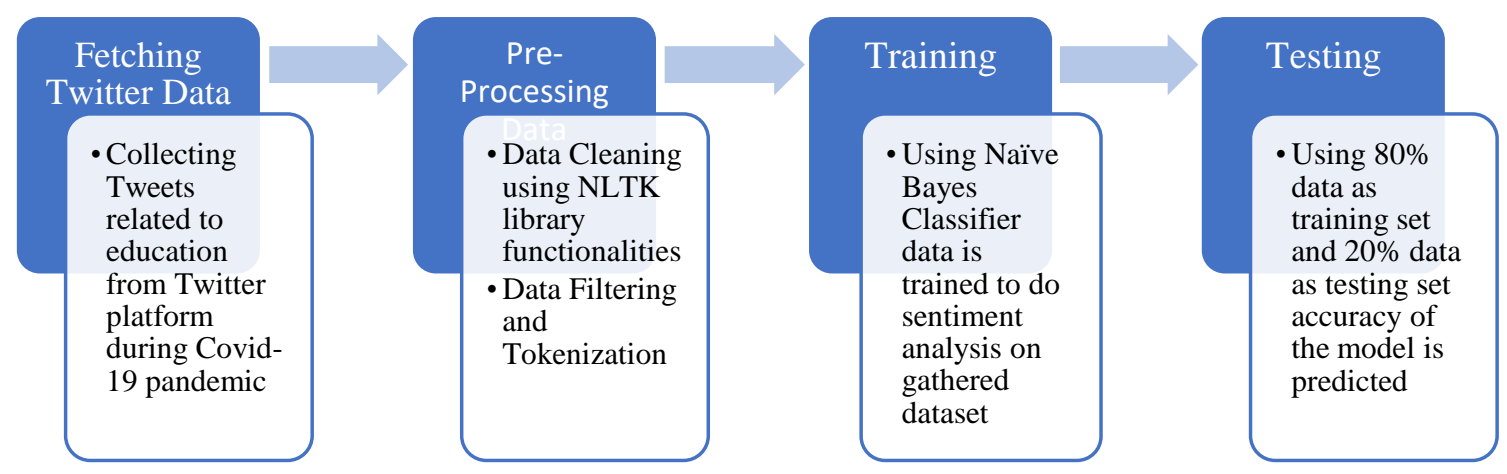

Figure 1. Shows the steps involved in the methodology

\subsection{Fetching Twitter Data}

The primary step is to gather the required data through extensively used social media platform Twitter. From mid-March almost every educational organization moved to remote functioning. So, based on that change, we gathered tweets from the months of March to July of the year 2020 for this study. To gather the data of this timeline, we used a customized get old tweets (GOT3) API from GitHub repository which uses URLlib to retrieve tweets from the twitter search engine as twitter API doesn't give free access to its data which is older than a week. We used certain specific keywords to query the twitter dataset which are observed as being generally used by people in their tweets to express their views about education. The keywords used are OnlineClasses, Virtual-Learning, Remote-Learning, Zoom-Classes and Distance-Learning. To avoid fetching tweets with text, which is not in English language, we have used Google's language detection library in Python named as langdetect and specified the preferred language as English [8]. Through the months of March to July, we have collected tweets from non-consecutive randomly chosen six dates of each month so that we could certainly identify the difference in public sentiments through each month from time to time. For every six days of a month, we have collected about minimum of 15 thousand tweets. Thus, we have a dataset of 91 thousand tweets from the months of March 2020 to July 2020. Having such a large dataset would help us gain better analysis.

\subsection{Pre-Processing the Data}

The data gathered in the above step contains raw tweets from twitter in which tweeters use different symbols, images, emoticons and links attached to their text message to express and support their context. Normally these raw tweets would have user handles, URL links, image 
International Journal of Artificial Intelligence and Applications (IJAIA), Vol.12, No.3, May 2021

links, punctuation marks, alpha numerals, numbers, emojis and other unnecessary symbols included with the text message [9]. Since tweets with such unnecessary links and symbols could bother the analysis procedure and affect the accuracy of the model, the data should be thoroughly cleaned. Using the "re" library of python we have performed data cleaning. After cleaning the data utilizing the functionalities of NLTK library data filtering and tokenization is done. Data filtering process involves removal of stop words such as prepositions and conjunctions from a tweet and tokenization is a process of splitting a tweet into tokens i.e., individual words [10]. Hence, raw tweets gathered are refined and each tweet is transformed into tokens which could be further passed for training procedure.

Table 1: Shows pre-processing of data with sample tweets

\begin{tabular}{|c|c|c|}
\hline Raw tweets & Clean tweets & Tokenized tweets \\
\hline $\begin{array}{l}\text { Yes indeed. A4: Moreover, } \\
\text { we should be in contact with } \\
\text { our students' parents to } \\
\text { supply them with the } \\
\text { necessary tools for remote } \\
\text { learning like comfortable } \\
\text { atmosphere, powerful access } \\
\text { to the internet, sufficient } \\
\text { devices. \#RemoteLearning } \\
\text { \#MSFTEduChat } \\
\text { \#MicrosoftEDU } \\
\text { \#TweetMeetEN } \\
\text { twitter.com/AbhilashaTochi } \\
\text { /status/125265743464199782 } \\
6 \text { Ixe2/x80\xa6""" }\end{array}$ & $\begin{array}{l}\text { Yes indeed Moreover we should be in } \\
\text { contact with our students parents to supply } \\
\text { them with the necessary tools for remote } \\
\text { learning like comfortable atmosphere } \\
\text { powerful access to the internet sufficient } \\
\text { devices } \\
\text { RemoteLearningMSFTEduChatMicrosoftED } \\
\text { UTweetMeetEN }\end{array}$ & $\begin{array}{l}\text { 'indeed', 'Moreover', } \\
\text { 'contact', 'students', } \\
\text { 'parents', 'supply', } \\
\text { 'necessary', 'tools', } \\
\text { 'remote', 'learning', } \\
\text { 'like', 'comfortable', } \\
\text { 'atmosphere', } \\
\text { 'powerful', 'access', } \\
\text { 'internet', 'sufficient', } \\
\text { 'devices', } \\
\text { 'RemoteLearning', } \\
\text { 'MSFTEduChat', } \\
\text { 'MicrosoftEDU', } \\
\text { 'TweetMeetEN' }\end{array}$ \\
\hline $\begin{array}{l}\text { So important to accept that } \\
\text { this is a difficult time for all } \\
\text { and very difficult for some. } \\
\text { As such keep things simple } \\
\text { and make sure expectations } \\
\text { are reasonable; This requires } \\
\text { discussing with students. } \\
\text { \#MicrosoftEdu \#MIEExpert } \\
\text { \#RemoteLearning } \\
\text { \#TweetMeetEn } \\
\text { twitter.com/robdunlopEDU/s } \\
\text { tatus/1252656849377046528 } \\
\text { Ixe2lx80|xa6' }\end{array}$ & $\begin{array}{l}\text { So important to accept that this is a difficult } \\
\text { time for all and very difficult for some As } \\
\text { such keep things simple and make sure } \\
\text { expectations are reasonable This requires } \\
\text { discussing with students MicrosoftEdu } \\
\text { MIEExpert } \\
\text { RemoteLearning } \\
\text { TweetMeetEn }\end{array}$ & $\begin{array}{l}\text { 'important', 'accept', } \\
\text { 'difficult', 'time', } \\
\text { 'difficult', 'keep', } \\
\text { 'things', 'simple', } \\
\text { 'make', 'sure', } \\
\text { 'expectations', } \\
\text { 'reasonable', } \\
\text { 'requires', } \\
\text { 'discussing', } \\
\text { 'students', } \\
\text { 'MicrosoftEdu', } \\
\text { 'MIEExpert', } \\
\text { 'RemoteLearning', } \\
\text { 'TweetMeetEn' }\end{array}$ \\
\hline $\begin{array}{l}\text { To me this is what } \\
\text { \#remotelearning has felt like! } \\
\text { Students plug in and out } \\
\text { where needed and suited their } \\
\text { needs. Redefining to me what } \\
\text { engagement looks, feels and } \\
\text { sounds like. Essentially this is } \\
\text { what my practice should have } \\
\text { looked like prior to } \\
\text { \#COVID19nz I guess \#nzhpe } \\
\text { pic.twitter.com/R81RTkTUoz } \\
\text { '" }\end{array}$ & $\begin{array}{l}\text { To me this is what remotelearning has felt like } \\
\text { Students plug in and out where needed and } \\
\text { suited their needs Redefining to me what } \\
\text { engagement looks feels and sounds like } \\
\text { Essentially this is what my practice should } \\
\text { have looked like prior COVIDnz I guess } \\
\text { nzhpe }\end{array}$ & $\begin{array}{l}\text { 'remotelearning', } \\
\text { 'felt', 'like', 'Students', } \\
\text { 'plug', 'needed', } \\
\text { 'suited', 'needs', } \\
\text { 'Redefining', } \\
\text { 'engagement', 'looks', } \\
\text { 'feels', 'sounds', 'like', } \\
\text { 'Essentially', } \\
\text { 'practice', 'looked', } \\
\text { 'like', 'prior', } \\
\text { 'COVIDnz', 'guess', } \\
\text { 'nzhpe' }\end{array}$ \\
\hline
\end{tabular}




\subsection{Training}

In our model to classify a tweet as positive or negative or as neutral we use a training dataset. A huge set of generic positive words and negative words files have been created. Using a bag of words function [11] we convert both the positive and negative word files into word dictionary and label each word of a positive word dictionary as positive and each word of a negative word dictionary as negative. Both the labelled positive and negative word dictionaries combined would serve as training data for our model.

Next, we use the generated training data with the Naïve Bayes classifier [12] in our model to generate a classifier. The tokenized tweets would be passed through this classifier and each token of tweet would be compared with training data and each token of tweet would be identified as positive or negative token. Then based on the possibility of positive or negative tokens in a tweet each tweet would have a positive probability and negative probability. Hence, a tweet with higher positive probability would be labelled as a positive tweet and a tweet with higher negative probability would be labelled as a negative tweet. For a tweet to be labelled as neutral tweet we have specified a neutral range of probability i.e. from 0.4 to 0.6 . When a tweet that has positive and negative probability of that specified range $(0.4-0.6)$ is found then the classifier would label that tweet as neutral.

Through this combination of word based training and Naïve Bayes classifier, we acquire the sentiment of each tweet amongst the gathered data. Thus, all the tweets are now labelled as either positive, negative or neutral.

\subsection{Testing}

The accuracy of this model depends on the positive and negative word files used as they are used to train the classifier and predict the tweet sentiment. Thus, the higher the number of words in the positive and negative word files, the better the training data and it would result in accurate labelling of tweets. To test the accuracy of the model described above to predict the sentiment of tweets, we split the labelled tweets into two data sets. $80 \%$ of the labelled tweets are used as training data and $20 \%$ of the labelled tweets are used as testing data. Applying the training and testing data sets to the multinomial Naïve Bayes classifier, we have an accuracy of $83.5 \%$ for our model.

\section{RESULTS AND ANALYSIS}

The research of performing sentiment analysis on twitter dataset of a total 91,701 tweets related to education during the Covid-19 phase from the months of March to July of the year 2020 has given an analysis result as follows

Table2: Shows percentage of the positive, negative and neutral tweets after performing sentiment analysis on the gathered dataset.

\begin{tabular}{|l|l|l|}
\hline Sentiment of tweet & Number of tweets & Percentage \\
\hline Positive tweets & 42,161 & 45.97 \\
\hline Negative tweets & 49,507 & 53.98 \\
\hline Neutral tweets & 33 & 0.03 \\
\hline
\end{tabular}


International Journal of Artificial Intelligence and Applications (IJAIA), Vol.12, No.3, May 2021

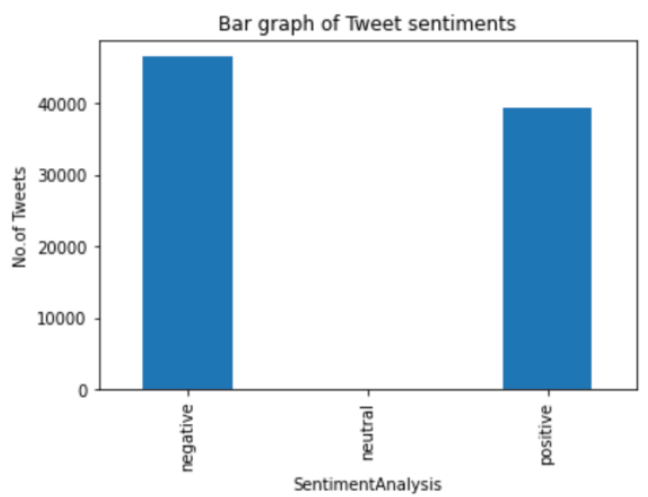

Figure 2: Bar graph representation of the classified tweets

Thus, the above analysis of dataset clearly indicates that the tweets collected include a very minimal number of neutral range tweets and the percentage of negative tweets is clearly higher than the positive tweets collected. To analyze the variation of tweets sentiments through each month, we have used matplotlib library of python to show a graphical visualization of positive, negative and neutral tweets collected per each of the six days from the months of March to July of the year 2020 as shown below

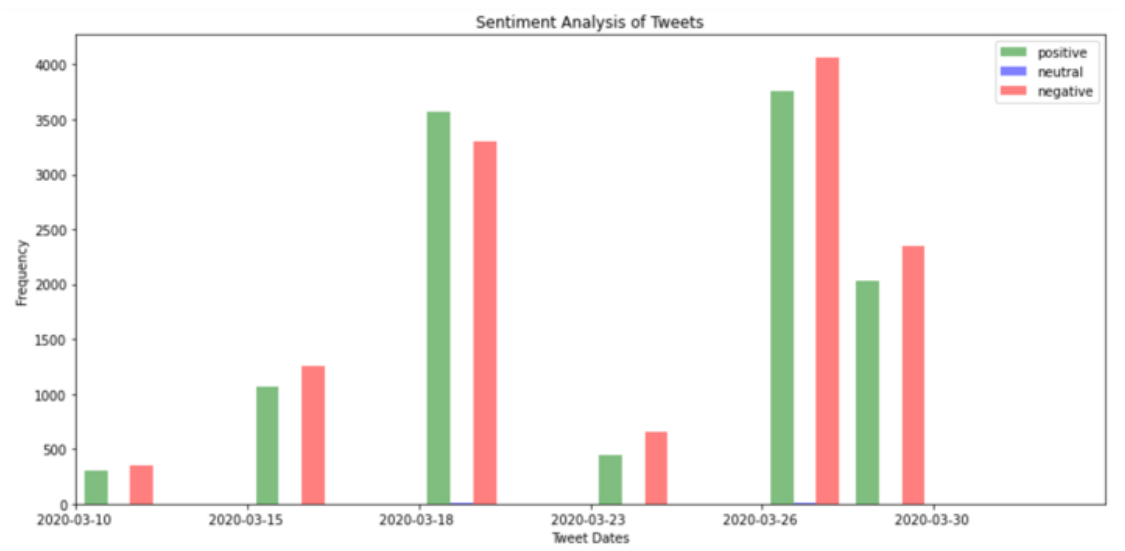

Figure 3: March 2020 tweets analysis

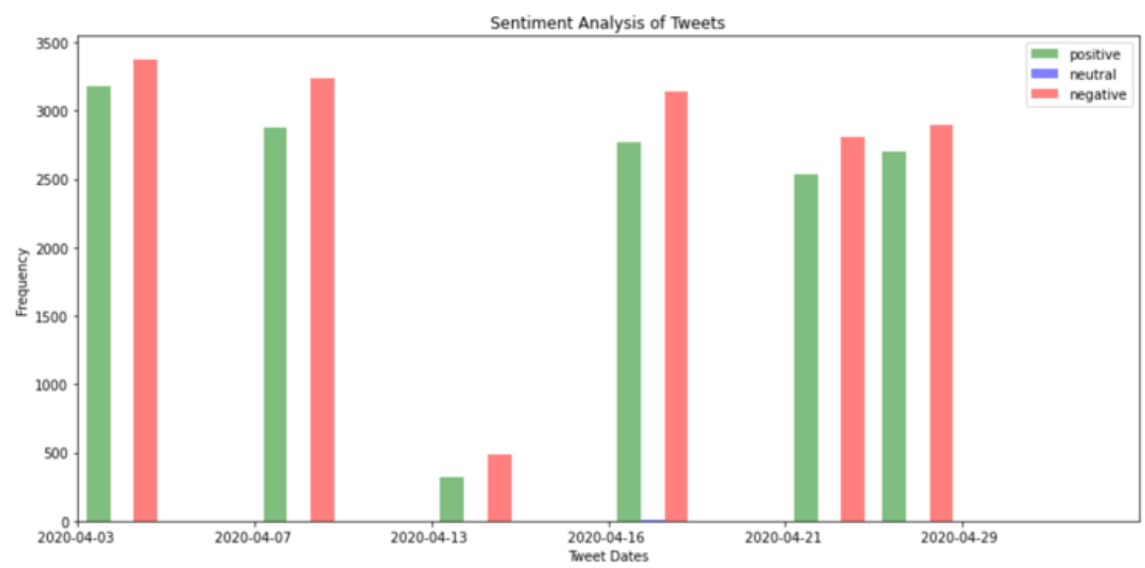

Figure 4: April 2020 tweets analysis 
International Journal of Artificial Intelligence and Applications (IJAIA), Vol.12, No.3, May 2021

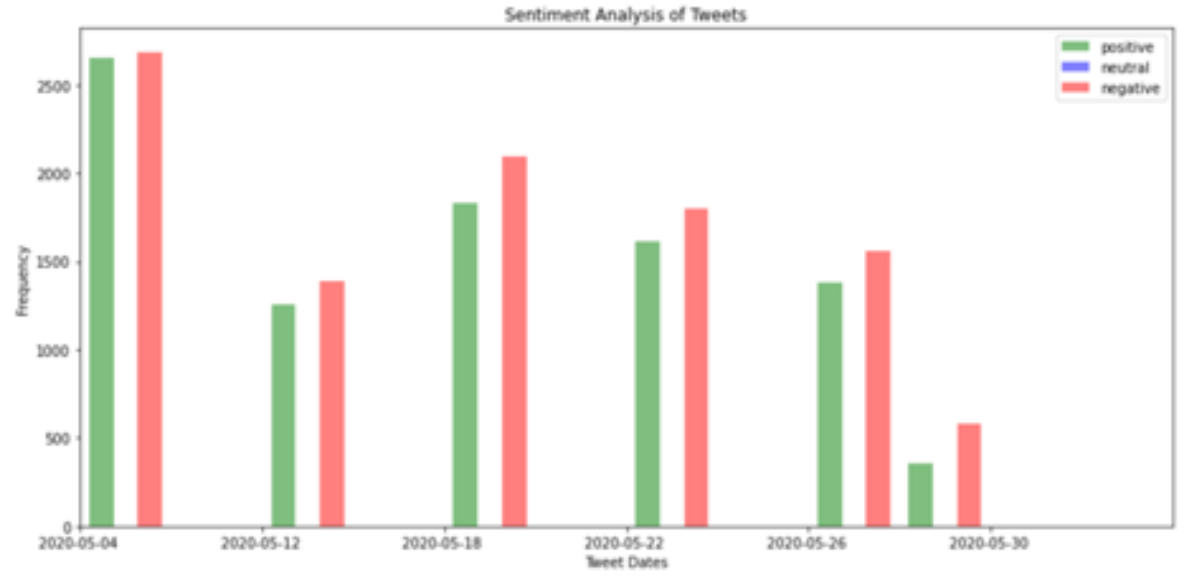

Figure 5: May 2020 tweets analysis

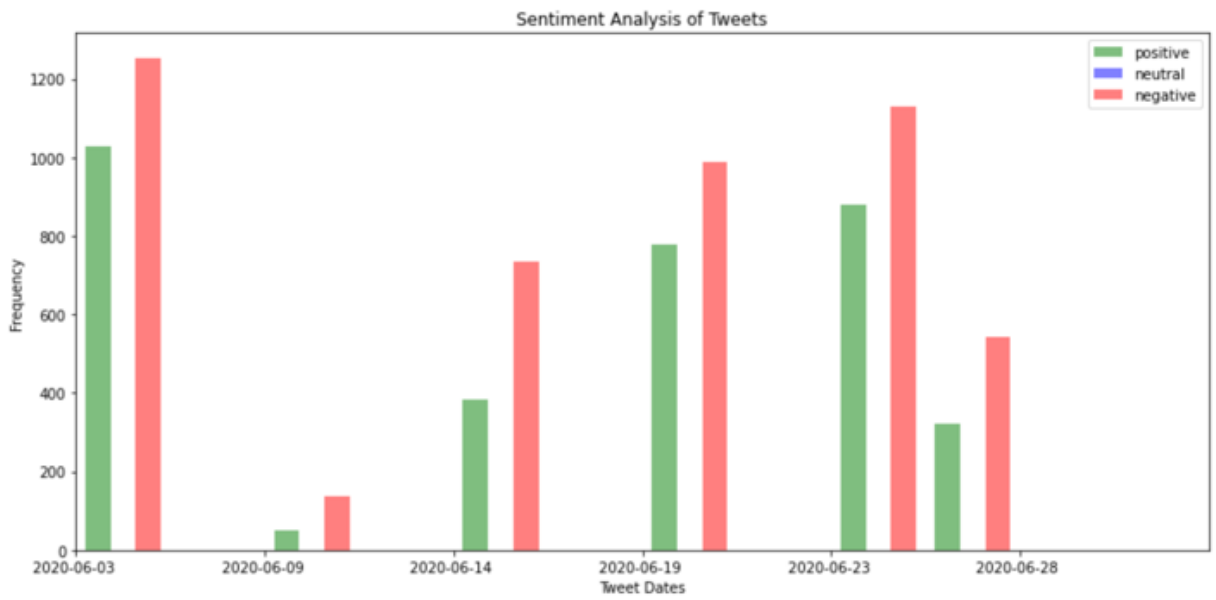

Figure 6: June 2020 tweets analysis

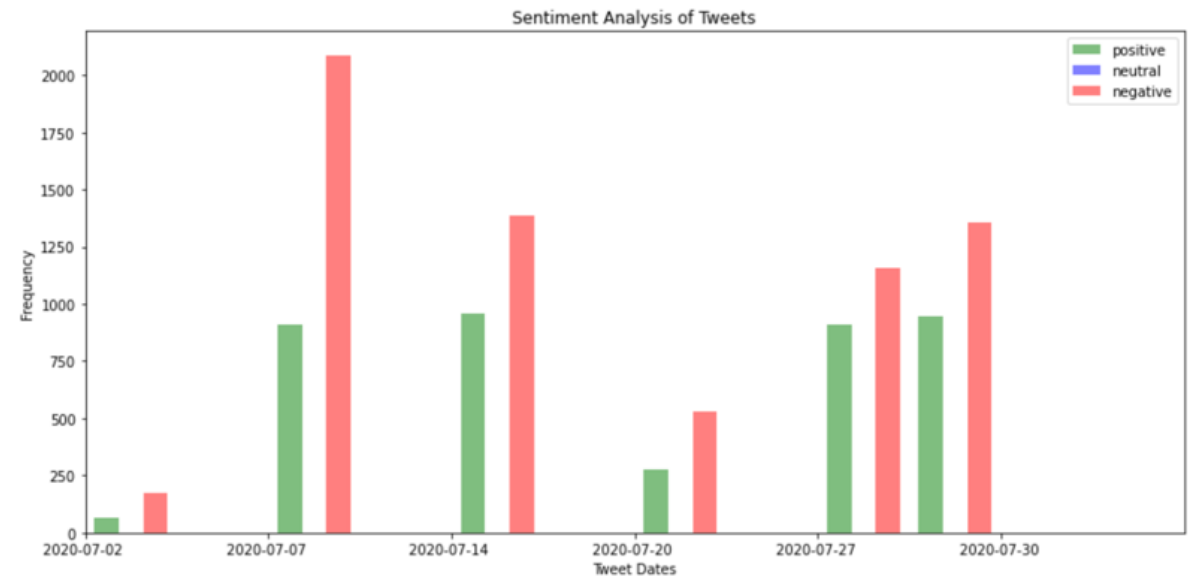

Figure 7: July 2020 tweets analysis 
International Journal of Artificial Intelligence and Applications (IJAIA), Vol.12, No.3, May 2021

From the graphs shown above, (Figures $3-7$ ), x-axis shows the dates on which tweets were collected and y-axis shows the number of tweets. Green bar in the picture indicates positive tweets and red bar indicates negative tweets collected on that day of each month. As the analysis of tweets progressed from the months of March to July, we could clearly see that there is a visible growth in negative tweets bar per day in comparison to the positive tweets bar. Neutral tweets bar isn't visible as they are very minimal but the space between bars representing positive and negative tweets indicate that each day there has been at least few neutral tweets tweeted.

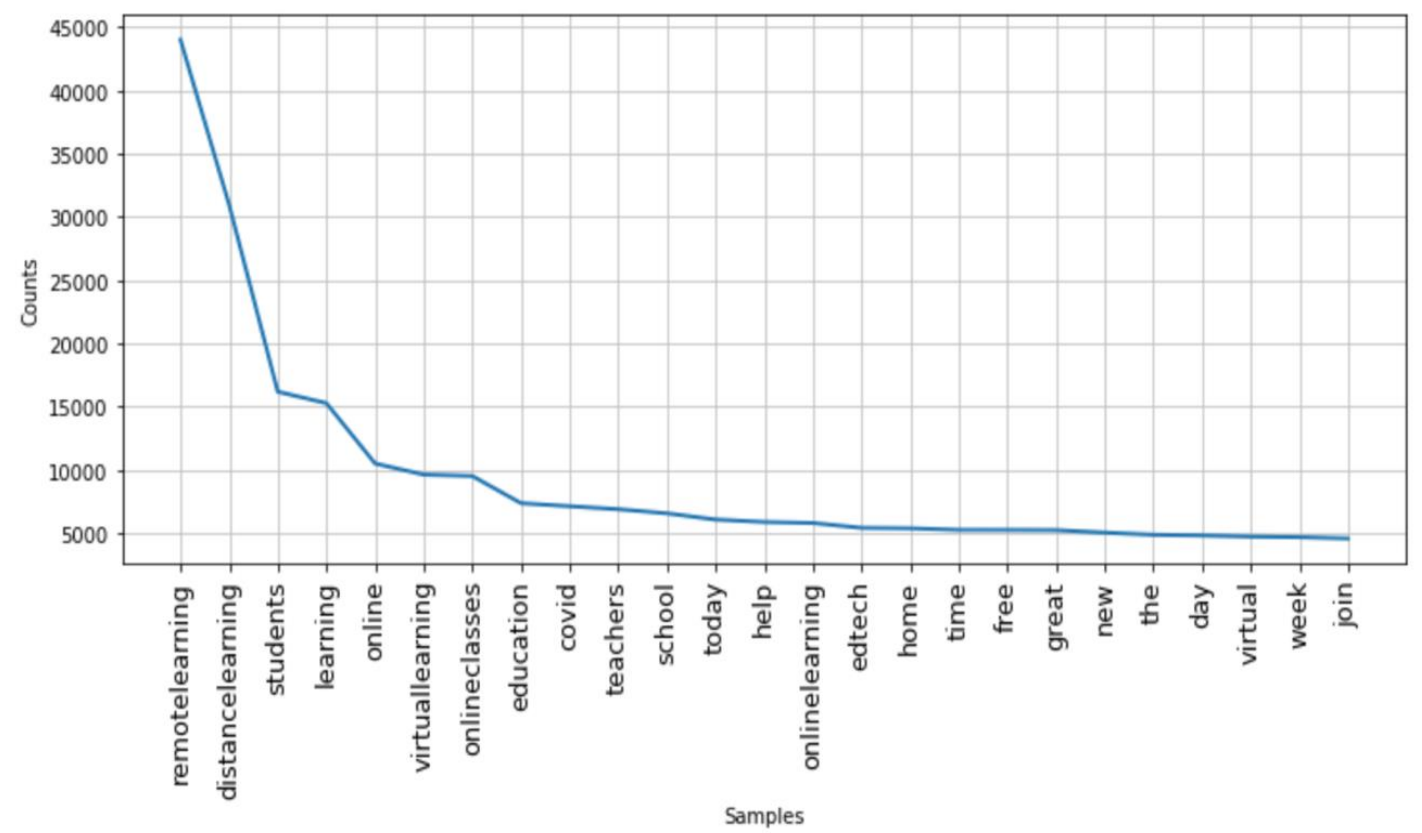

Figure 8: Shows the frequency distribution graph of top 25 most commonly used words in the dataset

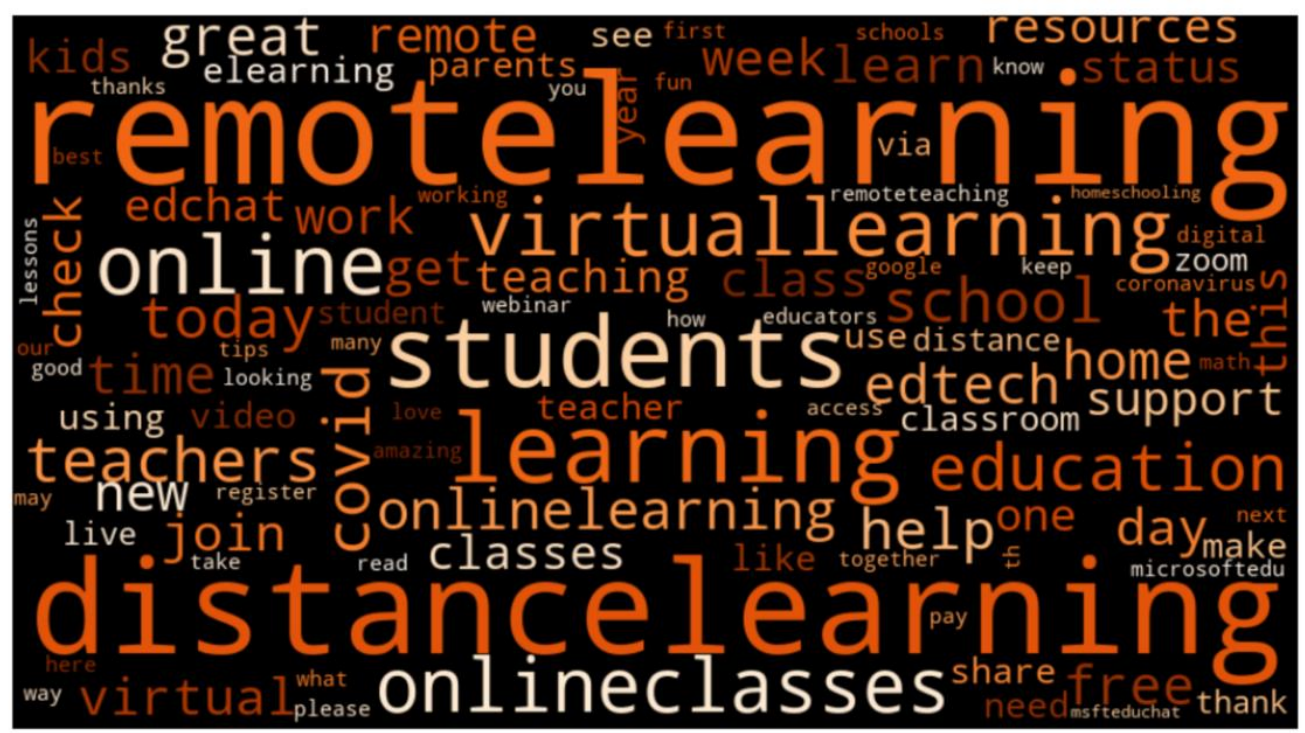

Figure 9: Shows Wordcloud of most commonly used words in the dataset 
International Journal of Artificial Intelligence and Applications (IJAIA), Vol.12, No.3, May 2021

Using features of word cloud library in python [13], we have plotted the frequency of the occurrence of most commonly used words in the gathered tweet dataset (Figure 8) and using word cloud picturization, the words that occurred most commonly in the tweets, are shown in Figure 9.

\section{Conclusion}

In this paper we have given an overview of the educational situation globally and effect of its shift to virtual learning and remote functioning during the Covid-19 pandemic. To study the effect of these dire changes in the education system and people's sentiment towards it, we have gathered related tweets and used a combination of word-based training and Naïve Bayes model on these tweets, we performed a sentiment analysis. Through this model, we have acquired sentiment of each tweet which reflects tweeter sentiment.

From the study results, we have seen that the number of tweets with negative sentiment is higher than the tweets with positive sentiment. Even though it seems safe to stay home and continue the education, there are clearly several other concerns being battled on daily basis to keep up with these changes in education system. There are many inconveniencies such as internet and tech issues, financial situation, and quality of education which have steered people to have negative sentiment towards the remote learning education being practiced now.

Further, we could implement this model and study people's sentiment across different countries independently and compare the difference in sentiments of the people about education. This could provide a perspective on this issue based on the geographical dimension. Also, we could try and include tweets in other languages as well for training and improve our model as a multilingual sentiment classifier.

\section{REFERENCES}

[1] "Impact of Covid-19 pandemic on education", from Wikipedia, the free encyclopedia. 2021.

[2] L. MO, L. J, Sheldenkar, S. PJ, S. W, Gupta R, and Y. Yang. Global Sentiments Surrounding the COVID-19 Pandemic on Twitter: Analysis of Twitter Trends. JMIR Public Health Surveillance. 2020.

[3] S. Burges and H. Henrik Sievertsen, "Schools, skills, and learning: The impact of COVID-19 on education" VoxEU \& CEPR. 2020.

[4] A. Pak and P. Paroubek. "Twitter as a Corpus for Sentiment Analysis and Opinion Mining". In Proceedings of the Seventh Conference on International Language Resources and Evaluation, pp.1320-1326. 2020.

[5] R. Go, B. Huang. "Twitter Sentiment Classification Using Distant Supervision" Stanford University, Technical Paper. 2009.

[6] A. Xie, I. Vovsha, O. Rambow, and R. Passonneau, "Sentiment Analysis of Twitter Data", In Proceedings of the ACL 2011Workshop on Languages in social media, pp. 30-38. 2011

[7] B. Pokharel, "Twitter Sentiment analysis during COVID-19 Outbreak in Nepal",SSRN Electronic Journal. January 2020.

[8] Jenny Lee, "Benchmarking Language Detection for NLP" Towards Data Science, 2020.

[9] A. Brahmananda Reddy, D.N. Vasundhara, P. Subhash, "Sentiment Research on Twitter Data" International Journal of recent Technology and Engineering (IJRTE). September 2019.

[10] Martin Pellarolo, "Naïve Bayes for Sentiment Analysis" Medium 2018.

[11] Yin Zhang, Rong Jin and Zhou, "Understanding bag-of-words model: a stastical framework" International Journal of Machine Learning and Cybernetics volume 1, 2010.

[12] Phyu Thwee, Yi Yi Aung, Cho Cho Lwin, "Naïve Bayes Classifier for sentiment analysis" International Journal of Creative and Innovative Research in All studies. January 2021.

[13] Florian Heimerl, Steffen Lohmann, Thomas Ertl "Word Cloud explorer: Text analytics based on word cloud" IEEE, 2014. 


\section{APPENDIX}

In this appendix we provide the links of libraries that we used.

A1. https://docs.python.org/3/library/

A2. https://github.com/Jefferson-Henrique/GetOldTweets-python

\section{ACKNOWLEDGEMENTS}

I would like to thank my advisors Dr. Ahmad Hadaegh and Dr. Yanyan Li who advised me and provided valuable comments in this work. I also like to thank my friends and my family for their support and encouragement during this time.

\section{AuTHORS}

Swetha Sree Cheeti was born in September 1994 in India. She completed her bachelor's in Computer Science from Jawaharlal Nehru Technological University (JNTU), Hyderabad, INDIA in 2016. She then moved to US in 2019 to pursue her Master of Science in computer science at California State University at San Marcos, California. She recently earned her graduation in Spring 2021 from CSUSM.

Yanyan Li received his Ph.D. degree from the University of Arkansas, Little Rock, in 2018. He is currently an Assistant Professor in the Department of Computer Science and Information Systems at California State University San Marcos. His research interests are in the areas of cybersecurity, machine learning, and mobile computing.

Dr. Hadaegh was born in Shiraz Iran. He moved to Canada in July 1983 and did his undergraduate work in computer science at University of Lethbridge in Alberta Canada. $\mathrm{He}$ moved to Winnipeg, Canada in 1988 to do his master and $\mathrm{PhD}$ at University of Manitoba in Computer Science. Dr. Hadaegh was hired by California State University San Marcos (CSUSM) in Fall 2002 and he has been working at CSUSM since then. His expertise is in Databases and Data Mining.

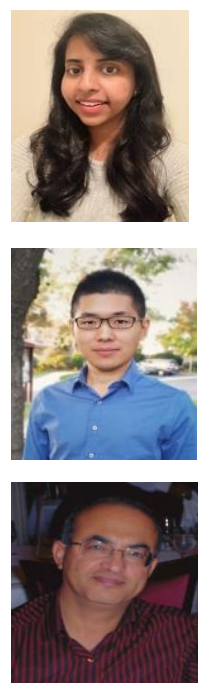

